This is the peer reviewed version of the following article: Sanchez-Delgado, G., Alcantara, J.M.A., Acosta, F.M., Martinez-Tellez, B., Amaro-Gahete, F.J., Merchan-Ramirez, E., Löf, M., Labayen, I., Ravussin, E. and Ruiz, J.R. (2020), Energy Expenditure and Macronutrient Oxidation in Response to an Individualized Nonshivering Cooling Protocol. Obesity, 28: 2175-2183., which has been published in final form at https://doi.org/10.1002/oby.22972. This article may be used for non-commercial purposes in accordance with Wiley Terms and Conditions for Use of Self-Archived Versions.

\title{
Energy expenditure and macronutrient oxidation in response to an individualized non-shivering cooling protocol
}

\author{
Guillermo Sanchez-Delgado ${ }^{\text {a,b }}$, Juan M.A. Alcantara ${ }^{a}$, Francisco M. Acosta a , Borja \\ Martinez-Tellez $^{\mathrm{a}, \mathrm{c}}$, Francisco J. Amaro-Gahete ${ }^{\mathrm{a}, \mathrm{d}}$, Elisa Merchan-Ramirez ${ }^{\mathrm{a}}$, Marie Löf \\ e,f , Idoia Labayen ${ }^{\mathrm{g}}$, Eric Ravussin ${ }^{\mathrm{b}}$, Jonatan R. Ruiz ${ }^{\mathrm{a}}$
}

${ }^{\text {a }}$ PROFITH (PROmoting FITness and Health through Physical Activity) Research Group, Sport and Health University Research Institute (iMUDS), Department of Physical Education and Sport, Faculty of Sport Sciences, University of Granada, Granada, Spain. ${ }^{\mathrm{b}}$ Pennington Biomedical Research Center, Baton Rouge, LA, United States of America.

${ }^{c}$ Department of Medicine, Division of Endocrinology, and Einthoven Laboratory for Experimental Vascular Medicine, Leiden University Medical Center, Leiden, Netherlands.

d Department of Medical Physiology. School of Medicine. University of Granada, Granada, Spain.

e Department of Biosciences and Nutrition, Karolinska Institutet, NOVUM, Huddinge, Sweden.

${ }^{\mathrm{f}}$ Department of Medical and Health Sciences, Faculty of Medicine and Health, Linköping University, 58183 Linköping, Sweden.

g Institute for Innovation and Sustainable Development in Food Chain (IS-FOOD), Navarra's Health Research Institute (IdiSNA), Department of Health Sciences, Public University of Navarra, 31006 Pamplona, Navarra, Spain.

Keyword: Thermogenesis; brown adipose tissue; metabolic rate; energy balance; obesity. Running title: Cold-induced thermogenesis and nutrient oxidation 
Contact info: Guillermo Sanchez-Delgado. Pennington Biomedical Research Center. 6400 Perkins Road, Baton Rouge, Louisiana, 70808, United States of America. E-mail: Guillermo.Sanchez@pbrc.edu; tel.: +1 (225)-763-2695.

Word count: 3051

Clinical trial registration: ClinicalTrials.gov ID: NCT02365129

Funding: This study was supported by the Spanish Ministry of Economy and Competitiveness via the Fondo de Investigación Sanitaria del Instituto de Salud Carlos III (PI13/01393) and PTA 12264-I, Retos de la Sociedad (DEP2016-79512-R) and European Regional Development Funds (ERDF), the Spanish Ministry of Education (FPU13/04365, FPU14/04172, and FPU15/04059), the Fundación Iberoamericana de Nutrición (FINUT), the Redes Temáticas de Investigación Cooperativa RETIC (Red SAMID RD16/0022), the AstraZeneca HealthCare Foundation, the University of Granada Plan Propio de Investigación 2016 -Excellence actions: Unit of Excellence on Exercise and Health (UCEES) - and Plan Propio de Investigación 2018 - Programa Contratos-Puente and Programa Perfeccionamiento de Doctores, the Junta de Andalucía, Consejería de Conocimiento, Investigación y Universidades (ERDF; ref. SOMM17/6107/UGR), and Fundación Alfonso Martín Escudero.

Disclosure: The authors declare no conflict of interest.

Author's contribution: GSD, JMAA, FMA, BMT, JRR: designed research; GSD, JMAA, FMA, BMT, FJAG, EMR: conducted research; GSD, JMAA: analyzed data; GSD, ML, ER, JRR: wrote paper; GSD, JRR: had primary responsibility for final content; GSD, JMAA, FMA, BMT, FJAG, EMR, ML, IL, ER, JRR: Discussed the results and approved the final version of the manuscript. 


\section{What is already known about this subject?}

- A decade ago, brown adipose tissue (BAT) was reported to be present and metabolically active in adult humans. Since then, non-shivering mild cold exposure has been considered a potential therapeutic tool in obesity management.

- Chronic exposure to non-shivering cold stimulus appears to improve energy metabolism and metabolic health in healthy individuals and people with obesity or type 2 diabetes mellitus.

\section{What are the new findings in your manuscript?}

- A mild cold exposure at a temperature adjusted to elicit maximum non-shivering thermogenesis induces a modest increase in energy expenditure, which is maintained constant during an hour.

- A metabolic shift on macronutrient oxidation rates to sustain cold-induced thermogenesis (CIT) was observed by which fat oxidation increased in parallel to a decrease in carbohydrate oxidation at the beginning of cold exposure. Later, an inverse tendency appeared by which carbohydrate oxidation increased and fat oxidation slightly decreased.

\section{How might your results change the direction of research or the focus of clinical practice?}

- This study suggests that mild cold exposure is not a feasible tool to induce negative energy balance in humans. However, pharmacological or nutraceutical continuous induction of maximum non-shivering thermogenesis might be a feasible target for weight loss.

- The observed shift on macronutrient oxidation rates may indicate that tissues playing a key role on CIT (i.e. BAT and muscles) could be different during the first stages of cold exposure than after 30 minutes of cold exposure. 


\section{ABSTRACT}

Objective: To describe the energy expenditure (EE) and macronutrient oxidation response to an individualized non-shivering cold exposure in young healthy adults.

Methods: Two different groups of 44 (Study 1: $22.1 \pm 2.1$ years old, $25.6 \pm 5.2 \mathrm{~kg} / \mathrm{m}^{2}, 34 \%$ men) and 13 young healthy adults (Study 2: $25.6 \pm 3.0$ years old, $23.6 \pm 2.4 \mathrm{~kg} / \mathrm{m}^{2}, 54 \%$ men) participated in this study. We measured resting EE (RMR) and macronutrient oxidation rates, by indirect calorimetry under fasting conditions, in a warm environment (30-minutes) and in mild cold conditions (65-minutes, wearing a water perfused cooling vest set at an individualized temperature adjusted to the individuals' shivering threshold).

Results: In study 1, EE increased in the initial stage of cold exposure and remained stable for the whole cold exposure $(\mathrm{P}<0.001)$. Mean CIT $(9.56 \pm 7.9 \mathrm{Kcal} / \mathrm{h})$ was $13.9 \pm 11.6$ \%RMR (Range: $-14.8 / 39.9 \%$ RMR). Carbohydrate oxidation decreased during the first 30 minutes of the cold exposure and later recovered up to the baseline values $(\mathrm{P}<0.01)$ in parallel to opposite changes in fat oxidation $(\mathrm{P}<0.01)$. Results were replicated in study 2 .

Conclusions: A 1-hour mild cold exposure individually adjusted to elicit maximum nonshivering thermogenesis induces a very modest increase in EE and a shift of macronutrient oxidation that may underlie a shift in thermogenic tissues activity. 


\section{INTRODUCTION}

Since brown adipose tissue (BAT) was shown to be present and metabolically active in adult humans in 2009 (1-4), cold-induced thermogenesis (CIT) has received considerable attention as a possible target for stimulating BAT and energy expenditure (EE), helping weight control and counteracting obesity (5). Indeed, several independent groups have reported that chronic exposure to non-shivering cold stimulus seems to enhance energy metabolism and metabolic health in healthy individuals (6) and people with obesity (7) or type 2 diabetes mellitus (8). Noteworthy, it remains unclear whether human BATrelated health-benefits are explained by increases in EE or by other mechanisms beyond energy balance $(9,10)$. Moreover, there is an open debate regarding the relative contribution of BAT to CIT, in comparison with other thermogenic tissues such as skeletal muscle or white adipose tissue (11).

CIT is produced by both non-shivering and shivering mechanisms (12). Shivering thermogenesis is the EE necessary to cover involuntary skeletal muscle contractions in response to cold, while non-shivering thermogenesis refers to the energy consuming process not depending on muscle contraction such as uncoupling respiration in brown adipocytes mitochondria (13). Although some have reported that shivering thermogenesis can increase EE up 5-fold above resting metabolic rate (RMR), its uncomfortable nature, together with the loss of motor coordination, makes it a poorly tolerated stimuli, and therefore, a non-plausible option to be used in clinical settings (13). On the other hand, non-shivering thermogenesis produces moderate increases of EE, and estimations report a range between 0 and $30 \%$ of the RMR in young healthy adults (13). Moreover, it has been argued that non-shivering thermogenesis may be comfortable enough to be considered a possible tool in the prevention and treatment of obesity (13). Of note, shivering and non-shivering thermogenesis do not appear to occur in sequential phases 
but more as parallel phenomenon (i.e. even at light cold stimulation shivering thermogenesis seems to contribute to CIT) (14), and its relative contribution to CIT can vary within-individuals (15).

There is a large inter-individual variation in non-shivering thermogenesis and cold tolerance. Therefore, to study the responses to mild cold exposure, there is a need to individualize the cold stimulus to the individuals' cold tolerance $(16,17)$. van der Lans et al. (16) proposed to first assess a shivering threshold (i.e. lowest external temperature without evoking externally observable and perceived shivering) as a reference point for adjusting the temperature at which an individual should be exposed. Although other methods have been proposed such as skin temperature clamping (18) or cold perception adjusting (19), the lowest tolerable temperature above the shivering threshold has been broadly accepted and used as a valid approach to induce BAT activation and CIT $(17,20-$ 23). Some studies have however shown that skeletal muscle thermogenesis takes place with very mild cold stimuli before observing shivering (14). As a result, the shivering threshold approach may not fully exclude the skeletal muscle shivering thermogenesis.

Research has traditionally spent more attention on the study of shivering thermogenesis (24), and important gaps remain regarding the human physiological responses to mild cold exposure in terms of EE and metabolic fuels selection (12). We hypothesized that a mild cold exposure designed to elicit non-shivering thermogenesis would exert light, but clinically meaningful if maintained in time, modifications on EE and metabolic fuels selection. Thus, the present study aimed to describe the EE and macronutrient oxidation response to an individualized non-shivering cold exposure in young healthy adults. 


\section{METHODS}

\section{Participants}

Two different cohorts took part in this study (table 1). For the first cohort (thereinafter called study 1), 63 participants (45 female) were included in the study, all of them being part of the ACTIBATE study (25). However, only 44 out of 63 were included in the statistical analyses (Figure 1, see the reasons below). Study 1 was conducted in October and November 2016. For study 2, 13 participants were recruited and evaluated between December 2017 and January 2018. Inclusion criteria in both studies were: $<35$ years old, report being healthy, non-smoker and not taking any medication, have had a stable body weight $(<3 \mathrm{Kg}$ change) during the past 3 months, and not regularly exposed to cold. The study and written informed consent considered the last revision of the Declaration of Helsinki and were approved by the Human Research Ethics Committee of the University of Granada ( $\left.\mathrm{n}^{\circ} 924\right)$ and of the Servicio Andaluz de Salud (Centro de Granada, CEIGranada).

We used a dual X-ray absorptiometry scan (Discovery Wi, Hologic, Inc., Bedford, MA, USA) to assess body composition, while a Seca scale and a stadiometer (model 799, Electronic Column Scale, Hamburg, Germany) were used to measure weight and height, respectively.

\section{Previous conditions to the study days}

Participants attended to the research center on two separate occasions. During the first visit, the individual's shivering threshold was assessed while during the second visit CIT was measured. They were asked to sleep as usual, to avoid both moderate ( 24 hours) and vigorous (48 hours) physical activity prior to the testing days, and to commute by motorized vehicle. 
In study 1, participants fasted for at least 6 hours; $9 \pm 3.7$ hours. Only those participants strictly following the fasting time indications (i.e. between 6 and 8 hours; $n=18$ ) were included in the macronutrient oxidation rate analyses $(26,27)$ (table 1$)$. In study 2 , participants were instructed to consume a standardized meal (35\% of estimated total EE; boiled rice, tomato sauce, and omelet) 10 hours before the CIT assessment. Moreover, they were instructed to collect all the urine from the standardized meal to the CIT assessment start (i.e. 10 hours urine production). Another urine sample was collected immediately after the CIT assessment.

\section{Shivering threshold test (day 1)}

The shivering threshold assessment methodology has been extensively described elsewhere $(23,28)$. In brief, after voiding, the participants dressed-up with standardized clothes (clothing insulation value: 0.20$)$. They then entered a warm room $\left(22.1 \pm 1.6^{\circ} \mathrm{C}\right)$ where they remained seated for 30 minutes, before entering a cold room $\left(19.8 \pm 0.5^{\circ} \mathrm{C}\right)$, where they dressed-up with a temperature-controlled water perfused cooling vest (Polar Products Inc., Ohio, USA). The vest circulating-water temperature was initially set at $16.6^{\circ} \mathrm{C}$ and was decreased every 10 minutes until $3.8^{\circ} \mathrm{C}$ was reached or until shivering occurred. Shivering was determined visually and self-reported. The vest circulating-water temperature when shivering started was considered as the shivering threshold.

\section{CIT and cold-induced macronutrient oxidation rates (day 2)}

Participants returned to the lab (the same time of day as before) 5-7 days (study 1) or 2 days (study 2) after the shivering threshold test. After voiding, they dressed in the same standardized clothes and entered a warm $\left(23.2 \pm 0.7^{\circ} \mathrm{C}\right)$ room. Before assessing EE, all participants reclined in a bed for 20 minutes. Later, their RMR was assessed over 30 minutes, following current methodological guidelines (29). Immediately after, the 
participants walked into the cold room $\left(19.7 \pm 0.4^{\circ} \mathrm{C}\right)$ and dressed in the cooling vest set at $4^{\circ} \mathrm{C}$ above the participant's shivering threshold. They laid in a reclined bed while the CIT assessment was carried out for two 30-minute periods, separated by a 5-minute pause. For assessing RMR and CIT, ventilatory gas exchange was collected by using a neoprene face-mask hooked up to a CCM Express or Ultima CardiO2 metabolic cart (Medgraphics Corp, Minnesota, USA) $(30,31)$. Flow, at the beginning of every test day, and gas analyzers, before every 30-minute assessment, were calibrated following the manufacturer's instructions.

In study 1, despite of the careful assessment of shivering threshold, 17 (16 women) out of 63 participants shivered during the CIT assessment. Those individuals were excluded from further statistical analysis. Moreover, 2 males were also excluded from the analyses for presenting respiratory exchange ratio (RER) values higher than 1.1 or lower than 0.7 in any measure point (29) (Figure 1).

\section{CIT and macronutrient oxidation rates estimation}

Oxygen consumption $\left(\mathrm{VO}_{2}\right)$ and carbon dioxide production $\left(\mathrm{VCO}_{2}\right)$ were downloaded from the metabolic cart software (Breeze Suite, 8.1.0.54 SP7) every minute. In all 30minute record period we discarded the first 5-minute record. We then selected the most stable 5-minute period among the remaining records (25 minutes) to be considered as the RMR, as previously described $(30,32)$. For calculating CIT, data was averaged every 5 minutes. In addition, we divided the CIT record into 4 parts and selected the most stable 5-minute period within every part. Finally, we calculated the area under the curve (trapezoidal rule) using the 4 selected 5-minute periods and the RMR, expressing it as a percentage of RMR. We have previously published that this method for data analyses resulted in more plausible estimations when compared to other methods for data analyses (33). The Weir's abbreviated equation was used to estimate EE (a) (34), while Frayn's 
equation (35) (not taking into account any urinary nitrogen in study 1) were used for estimating carbohydrates (CHOox) (b) and fat oxidation (FATox) (c).

(a) $E E(\mathrm{Kcal} / \mathrm{min})=3.941 \times V O_{2}(1 / \mathrm{min})+1.106 \times V C O_{2}(1 / \mathrm{min})-2.17 \times \mathrm{N}(\mathrm{g} / \mathrm{min})$

(b) $C H O o x(\mathrm{~g} / \mathrm{min})=-3.21 \times V O_{2}(1 / \mathrm{min})+4.55 \times V C O_{2}(1 / \mathrm{min})-2.87 \times \mathrm{N}(\mathrm{g} / \mathrm{min})$

(c) $\operatorname{FATox}(\mathrm{g} / \mathrm{min})=1.67 \times V O_{2}(1 / \mathrm{min})-1.67 \times V C O_{2}(1 / \mathrm{min})-1.92 \times \mathrm{N}(\mathrm{g} / \mathrm{min})$

In study 2, we measured total urine volume and urea concentration in both urine samples. Thereafter, we estimated nitrogen urine levels from the urea concentration (Spinreact, Catalogue No. 283-17) following the equation "N(g/l)=0.0065* Urea(mg/dl $)+1.2598 "$ obtained from a linear regression including urea and nitrogen measured concentrations (kjeldahl method (36)) from an independent cohort of young healthy adults $(n=19,16$ women; $21.87 \pm 2.05$ years old; $\left.24.87 \pm 3.71 \mathrm{~kg} / \mathrm{m}^{2}\right)$. Protein oxidation $(\mathrm{g} / \mathrm{min})$ was calculated as $\mathrm{N}(\mathrm{g} / \mathrm{min}) \times 6.25(37)$.

\section{Statistical analysis}

Repeated-measures analyses of variance (ANOVA), with Bonferroni post-hoc corrections, were used to test differences in EE, CHOox and FATox across time. Paired t-test were used to compare protein oxidation before and during the cold stimulus.

The analyses were conducted using the Statistical Package for Social Sciences (SPSS, v. 21.0, IBM SPSS Statistics, IBM Corporation). Figures were created using Graph Pad Prism (GraphPad Software, v. 8.3.1, CA, USA). The level of significance was set at $<0.05$. 


\section{RESULTS}

Mild cold exposure significantly increased $\mathrm{EE}(\mathrm{P}<0.001$; Figure $2 \mathrm{~A})$ from the beginning of cold-exposure and remained stable from that moment till the end of the mild cold

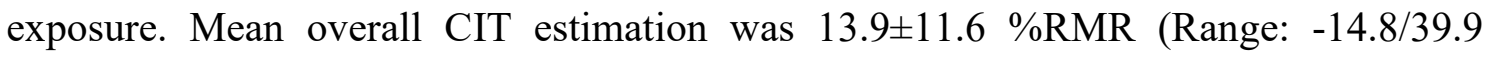
$\%$ RMR, Figure 2B). When translated to cumulative EE (i.e. the total amount of energy above the RMR expended during the whole 65-minute cold exposure), it resulted in $9.56 \pm 7.9$ Kcal.

CHOox decreased after 30 minutes of mild cold exposure $(\mathrm{P}=0.001)$, although its level returned to the baseline after 40 minutes of mild cold exposure (Figure 2F). The peak FATox was observed at 30 minutes of cold exposure $(\mathrm{P}<0.001$; Figure $2 \mathrm{~F})$. FATox progressively increased until minute 30 , and later on, there was a decrease. All results were similar in men and women, and in individuals with $\mathrm{BMI}<25 \mathrm{~kg} / \mathrm{m}^{2}$ and $\mathrm{BMI}>25 \mathrm{~kg} / \mathrm{m}^{2}$ (data not shown). Mean overall CI CHOox was $-7.3 \pm 22.5 \%$ basalCHOox (Range: $-36.4 / 34.31 \%$ basalCHOox) and mean overall CI FATox was 53.6 \pm 90.9 \%basalFATox (Range: -33.9/382.1\% basalFATox).

Figure 3 shows EE and macronutrient oxidation rates during a mild cold exposure in the study 2. Cold exposure increased EE as in study 1 ( $\mathrm{P}>0.001$; Figure $3 \mathrm{~A})$. The effect of cold exposure on both CHOox and FATox (Figure 3F) was also similar to study 1. Finally, Figure 4 shows the estimated protein oxidation before (10 hours) and during the mild cold exposure in study 2. Mild cold exposure significantly increased protein oxidation $(0.91 \pm 0.38 \mathrm{~g} / \mathrm{min}$ during the cold exposure vs. $0.54 \pm 0.29 \mathrm{~g} / \mathrm{min}$ for the $10 \mathrm{~h}$ preceding cold exposure; $\mathrm{P}=0.005$ ). 


\section{DISCUSSION}

We show that a 1 hour individualized mild cold exposure produced a moderate increase in energy expenditure $(9.56 \pm 7.9 \mathrm{Kcal} / \mathrm{h})$ in young healthy adults. Interestingly, we observed a metabolic shift in time for sustaining CIT. CHOox decreased during the first 30 minutes of the cold exposure and recovered up to the baseline values at the end of the cold exposure. In contrast, FATox continuously increased during the first 30 minutes, and decreased in the second part of the cold exposure. Of note is that these results were replicated in two independent studies which further reinforce the findings. Our results show a modest EE increase during mild cold exposure which concurs with previous studies (12). Our data also show a previously unreported shift in the nutrient oxidation rates for sustaining the CIT during the first phase of mild cold exposure (first 30 minutes). This finding might have important implications for the understanding of physiological processes underlying human non-shivering thermogenesis.

\section{Mild cold exposure induces moderate increases in energy expenditure}

In study 1, some individuals showed negative values of CIT (i.e. lower EE in cold than in RMR) while others experienced a 39\% increase over RMR, which concurs with the huge inter-individual variability $(38,39)$ reported in previous studies. Whether nonshivering cold exposure induces a stable energy expenditure change from the beginning of the exposure is unclear (40). We observed that when applying an individualized cooling protocol at a temperature near to the shivering threshold, maximum non-shivering thermogenesis appears to be elicited from the beginning of the cold exposure. This constant thermogenic rate concurs with other studies analyzing the EE elicited by shivering $(41,42)$. On the other hand, it is not clear whether the magnitude of nonshivering thermogenesis is able to induce a significant negative energy balance $(14,43)$. We observed that even when applying a protocol designed to elicit maximum non- 
shivering thermogenesis, all participants presented an EE below 1.4 metabolic equivalent (MET, i.e. $40 \%$ of RMR increase). Taking into account that even a very low intensity exercise, such as walking, can elicit 2-3 METs, together with the fact that cold exposure may induce an hyperphagic response (44), it seems that cold exposure at temperatures eliciting low shivering and maximum non-shivering thermogenesis are not an efficient stimuli to induce negative energy balance. It should also be noted that this mild cold stimulus produces a considerable burden and discomfort for participants, despite only 9.56 \pm 7.9 Kcal were burned (above the RMR) after an hour at this uncomfortable situation. Nonetheless, if maximum non-shivering thermogenesis were induced by pharmacological or nutraceutical agents $(45,46)$, instead of cold exposure, and were continuously elicited during $24 \mathrm{~h}$, our data would translate into $212 \pm 175 \mathrm{Kcal} /$ day. Importantly, recent studies using radiotracers for the quantification of BAT energy expenditure have estimated that, if maximally and continually activated, human BAT could account for as much as $\approx 15$ $\mathrm{Kcal} /$ day $(20,47,48)$. This is less than $10 \%$ of the $24 \mathrm{~h}$ CIT estimation made in our study, which suggest that tissues other than BAT (e.g. skeletal muscle) may represent better targets for stimulating non-shivering thermogenesis in humans (5). Nonetheless, alternative mechanisms beyond energy balance $(9,10)$ have been suggested to mediate the health promoting effects of BAT and chronic cold exposure (6-8). Therefore, mild cold exposure and/or pharmacological BAT activation or recruitment might not be discarded as possible therapeutic target including insulin sensitizing effects.

\section{Mild cold exposure induced a shift on fuel oxidation}

We observed a marked shift in macronutrient oxidation to sustain the thermogenic rate. Previous studies have reported similar metabolic shifts at a constant thermogenic rate in response to cold (in shivering conditions) $(12,41,42)$, however, we observed a decrease in CHOox, which has not been reported before. Preserving muscle glycogen is considered 
to have a profound impact on cold endurance, and therefore on cold survival (12). Consequently, fatty acids are the most sustainable fuel for thermogenic purposes. FATox is the predominant substrate for both non-shivering and shivering thermogenesis (12). Therefore, it is biologically plausible that a shift to FATox is produced when thermogenic needs are not maximized, such as at the beginning of the cold exposure.

BAT is not the only contributor responsible for non-shivering thermogenesis in humans. Skeletal muscle possibly contributes to non-shivering thermogenesis even to a larger extent $(14,20)$. Although both BAT and skeletal muscle preferentially use fatty acids as energy fuel in non-shivering situations, BAT is probably more FATox-preferential, as more than $90 \%$ of its energy consumption relies on fat oxidation $(49,50)$, while skeletal muscle presents a more balanced nutrient uptake (12). Therefore, it is plausible to speculate with the possibility of BAT being the main contributor to CIT at initial stages of mild cold exposure while muscle contribution to CIT would increase progressively, therefore balancing the contribution of CHOox and FATox. This hypothesis is supported by recent studies showing that BAT is rapidly activated upon cold exposure and seems to stabilize after 35 minutes of cold exposure (51-53). Moreover, we previously reported a high prevalence of BAT in study $1(88 \%$ BAT positive, BAT volume $=94.4 \pm 59.6 \mathrm{ml})$, measured by static ${ }^{18} \mathrm{~F}$-Flurodeoxyglucose PET/CT scan after 2 hours of personalized cold exposure, in study 1 participants $(23,54)$; therefore BAT thermogenesis was likely induced during the mild cold exposure. New studies examining the contribution of both tissues, BAT and skeletal muscle, from the very beginning of the cold exposure are needed to confirm such hypothesis.

Protein oxidation was only assessed in study 2, in which we observed a significant increase in response to mild cold exposure. A recent study in both mice and humans showed that upon cold exposure, BAT significantly increases the uptake and oxidation of 
branched-chain amino acids (BCAA) (55). Importantly, blunting BCAA oxidation in BAT significantly affected BAT thermogenic capacity in mice (55). Therefore, the increase of protein oxidation observed in our study might be explained by the increased uptake and oxidation of BCAA in BAT. Such statement is quite speculative since we did not specifically measure BCAA oxidation in study 2 . Further studies are needed to test whether there is an association between BAT activity and cold-induced protein oxidation in humans.

In agreement with previous studies (12), we observed a considerable high inter-individual variability in cold-induced macronutrient oxidation rates. In human studies, different patterns of shivering (i.e. muscle recruitment) explain most of the inter-individual variability in cold-induced macronutrient oxidation rates $(12,41,42)$. Since the protocol we applied is considered to result in low shivering contribution to CIT, different patterns of shivering may not explain such a large inter-individual variability. Alternatively, interindividual differences on tissues (BAT, skeletal muscle, and white adipose tissue) relative contribution to CIT (i.e. proportion of CIT being produce by each tissue) might partially explain such a high inter-individual difference (5).

\section{Limitations}

In study 1, carbohydrates and fat oxidation rates were calculated without considering protein oxidation, since we did not measure urine nitrogen excretion. In addition, in study 1, we did not strictly control fasting period and previous meal content. However, we performed a confirmatory study (i.e. study 2) analyzing protein oxidation and strictly controlling fasting time, previous meal content and similar results were found. Secondly, the visually detected or self-reported shivering is likely to occur after electrical muscle activity (40). Therefore, the absence of electromyographical recording might have underestimated the shivering threshold temperature. Moreover, despite we excluded 
participants presenting detectable shivering during the CIT assessment, we cannot discard that non-visually detectable nor self-noted shivering thermogenesis was present (14). Nonetheless, it is probable that non-shivering thermogenesis was the predominant form of CIT in the included participants. In addition, it should be noted that the study sample was entirely composed of young (18-32 years) healthy individuals, and thus the results cannot be generalized to older or unhealthy individuals. Finally, it should be noted that the reliability of the indirect calorimeters used in this study was not optimal (31). This probably explains the presence of non-physiological values obtained in 2 out of our 63 participants enrolled in study 1 . 


\section{CONCLUSIONS}

A mild cold exposure at a temperature adjusted to elicit maximum non-shivering thermogenesis induces a very modest increase in EE ( $<40 \% \mathrm{RMR} ; \approx 1.4 \mathrm{METs})$, which is maintained constant during an hour. The cumulated cold-induced energy expenditure over an hour of mild cold exposure $(9.6 \pm 7.9 \mathrm{Kcal} / \mathrm{h})$ is probably insufficient to induce a negative energy balance in humans. However, we cannot exclude that sustained induction of maximum non-shivering cold-induced thermogenesis $(212 \pm 175 \mathrm{Kcal} /$ day $)$, which might be achieved by pharmacological or nutraceutical agents, can be a feasible strategy for weight loss. Interestingly, we found a metabolic shift on macronutrient oxidation rates to sustain CIT by which FATox increased in parallel to a decrease in CHOox at the beginning of cold exposure. Later, an inverse tendency appeared by which CHOox increased and FATox slightly decreased. This may indicate that tissues playing a key role in CIT (i.e. BAT and muscles) could be different during the first stages of cold exposure than after 30 minutes of cold exposure. 


\section{ACKNOWLEDGMENT}

This study is part of a Ph.D. Thesis conducted in the Biomedicine Doctoral Studies of the University of Granada, Spain. We are grateful to Carmen Sainz-Quinn and Jacqueline Fox for assistance with the English language. 


\section{REFERENCES}

1. Cypess AM, Lehman S, Williams G, et al. Identification and importance of brown adipose tissue in adult humans. $N$ Engl J Med 2009;360:1509-17.

2. Saito M, Okamatsu-Ogura Y, Matsushita M, et al. High incidence of metabolically active brown adipose tissue in healthy adult humans: effects of cold exposure and adiposity. Diabetes 2009;58:1526-31.

3. van Marken Lichtenbelt WD, Vanhommerig JW, Smulders NM, et al. Coldactivated brown adipose tissue in healthy men. $N$ Engl J Med 2009;360:1500-8.

4. Virtanen KA, Lidell ME, Orava J, et al. Functional brown adipose tissue in healthy adults. $N$ Engl J Med 2009;360:1518-25.

5. Palmer BF, Clegg DJ. Non-shivering thermogenesis as a mechanism to facilitate sustainable weight loss. Obes Rev 2017:1-13.

6. Lee $\mathrm{P}$, Smith $\mathrm{S}$, Linderman $\mathrm{J}$, et al. Temperature-acclimated brown adipose tissue modulates insulin sensitivity in humans. Diabetes 2014;177:1-59.

7. Hanssen MJW, van der Lans AAJJ, Brans B, et al. Short-term Cold Acclimation Recruits Brown Adipose Tissue in Obese Humans. Diabetes 2016;65:1179-89.

8. Hanssen MJW, Hoeks J, Brans B, et al. Short-term cold acclimation improves insulin sensitivity in patients with type 2 diabetes mellitus. Nat Med $2015 ; 21: 863-5$.

9. Peirce V, Vidal-Puig A. Regulation of glucose homoeostasis by brown adipose tissue. lancet Diabetes Endocrinol 2013;1:353-60.

10. Villarroya F, Cereijo R, Villarroya J, Giralt M. Brown adipose tissue as a 
secretory organ. Nat Rev Endocrinol 2017;13:26-35.

11. Carpentier AC, Blondin DP, Virtanen KA, Richard D, Haman F, Turcotte ÉE. Brown Adipose Tissue Energy Metabolism in Humans. Front Endocrinol (Lausanne) 2018;9:1-21.

12. Blondin DP, Tingelstad HC, Mantha OL, Gosselin C, Haman F. Maintaining thermogenesis in cold exposed humans: Relying on multiple metabolic pathways. Compr Physiol 2014;4:1383-1402.

13. van Marken Lichtenbelt WD, Kingma B, van der Lans A, Schellen L. Cold exposure--an approach to increasing energy expenditure in humans. Trends Endocrinol Metab 2014;25:165-7.

14. Blondin DP, Labbé SM, Phoenix S, et al. Contributions of white and brown adipose tissues and skeletal muscles to acute cold-induced metabolic responses in healthy men. $J$ Physiol 2015;593:701-14.

15. Blondin DP, Daoud A, Taylor T, et al. Four-week cold acclimation in adult humans shifts uncoupling thermogenesis from skeletal muscles to BAT. J Physiol 2016;6:2099-2113.

16. van der Lans A a JJ, Wierts R, Vosselman MJ, Schrauwen P, Brans B, van Marken Lichtenbelt WD. Cold-activated brown adipose tissue in human adults: methodological issues. Am J Physiol Regul Integr Comp Physiol 2014;307:R10313.

17. Chen KY, Cypess AM, Laughlin MR, et al. Brown Adipose Reporting Criteria in Imaging STudies (BARCIST 1.0): Recommendations for Standardized FDGPET/CT Experiments in Humans. Cell Metab 2016;24:210-222. 
18. Blondin DP, Tingelstad HC, Noll C, et al. Dietary fatty acid metabolism of brown adipose tissue in cold-acclimated men. Nat Commun 2017;8:14146.

19. Coolbaugh CL, Bush EC, Galenti ES, Welch EB, Towse TF. An Individualized, Perception-Based Protocol to Investigate Human Physiological Responses to Cooling. Front Physiol 2018;9:195.

20. U Din M, Raiko J, Saari T, et al. Human brown adipose tissue [(15)O]O2 PET imaging in the presence and absence of cold stimulus. Eur J Nucl Med Mol Imaging 2016;43:1878-86.

21. Bakker LEH, Boon MR, van der Linden $\mathrm{R}$ a $\mathrm{D}$, et al. Brown adipose tissue volume in healthy lean south Asian adults compared with white Caucasians: a prospective, case-controlled observational study. lancet Diabetes Endocrinol $2014 ; 2: 210-7$.

22. Leitner BP, Huang S, Brychta RJ, et al. Mapping of human brown adipose tissue in lean and obese young men. Proc Natl Acad Sci U S A 2017;114:6-11.

23. Martinez-Tellez B, Sanchez-Delgado G, Garcia-Rivero Y, et al. A new personalized cooling protocol to activate brown adipose tissue in young adults. Front Physiol 2017;8:1-10.

24. Haman F, Blondin DP. Shivering thermogenesis in humans: Origin, contribution and metabolic requirement. Temperature 2017;8940:1-10.

25. Sanchez-Delgado G, Martinez-Tellez B, Olza J, et al. Activating brown adipose tissue through exercise (ACTIBATE) in young adults: Rationale, design and methodology. Contemp Clin Trials 2015;45:416-25.

26. Jeukendrup AE, Wallis GA. Measurement of substrate oxidation during exercise 
by means of gas exchange measurements. Int J Sport Med Suppl 2005;26.

27. Venables MC, Achten J, Jeukendrup AE. Determinants of fat oxidation during exercise in healthy men and women: a cross-sectional study. $J$ Appl Physiol 2005;98:160-167.

28. Martinez-Tellez B, Sanchez-Delgado G, Acosta FM, et al. Differences between the most used equations in BAT-human studies to estimate parameters of skin temperature in young lean men. Sci Rep 2017;7:10530.

29. Fullmer S, Benson-Davies S, Earthman CP, et al. Evidence analysis library review of best practices for performing indirect calorimetry in healthy and noncritically ill individuals. J Acad Nutr Diet 2015;115:1417-1446.e2.

30. Sanchez-Delgado G, Alcantara JMA, Ortiz-Alvarez L, et al. Reliability of resting metabolic rate measurements in young adults: Impact of methods for data analysis. Clin Nutr 2018;37:1618-1624.

31. Alcantara JMA, Sanchez-Delgado G, Martinez-Tellez B, Merchan-Ramirez E, Labayen I, Ruiz JR. Congruent validity and inter-day reliability of two breath by breath metabolic carts to measure resting metabolic rate in young adults. Nutr Metab Cardiovasc Dis 2018.

32. Alcantara JMA, Sanchez-Delgado G, Amaro-Gahete FJ, Galgani JE, Ruiz JR. Impact of the Method Used to Select Gas Exchange Data for Estimating the Resting Metabolic Rate, as Supplied by Breath-by-Breath Metabolic Carts. Nutrients 2020;12:4-6.

33. Sanchez-Delgado G, Alcantara JMA, Acosta FM, et al. Estimation of nonshivering thermogenesis and cold-induced nutrient oxidation rates: Impact of 
method for data selection and analysis. Clin Nutr 2019;38:2168-2174.

34. Weir JBDB. New methods for calculating metabolic rate with special reference to protein metabolism. $J$ Physiol 1949;109:1-9.

35. Frayn KN. Calculation of substrate oxidation rates in vivo from gaseous exchange. J Appl Physiol 1983;55:628-34.

36. Kjeldahl J. Neue Methode zur Bestimmung des Stickstoffs in organischen Körpern. Fresenius’ Zeitschrift für Anal Chemie 1883;22:366-382.

37. Elia M, Livesey G. Energy expenditure and fuel selection in biological systems: the theory and practice of calculations based on indirect calorimetry and tracer methods. World Rev Nutr Diet 1992;70:68-131.

38. van Marken Lichtenbelt WD, Daanen H a M. Cold-induced metabolism. Curr Opin Clin Nutr Metab Care 2003;6:469-475.

39. van Marken Lichtenbelt WD, Schrauwen P, van de Kerckhove S, WesterterpPlantenga MS. Individual variation in body temperature and energy expenditure in response to mild cold. Am J Physiol Metab 2002;282:E1077-E1083.

40. Acosta FM, Martinez-Tellez B, Sanchez-Delgado G, et al. Physiological responses to acute cold exposure in young lean men. PLoS One 2018;13:e0196543.

41. Haman F, Peronnet F, Kenny GP, et al. Effects of carbohydrate availability on sustained shivering I. Oxidation of plasma glucose, muscle glycogen, and proteins. J Appl Physiol 2004;96:32-40.

42. Haman F, Legault SR, Rakobowchuk M, Ducharme MB, Weber J-M. Effects of carbohydrate availability on sustained shivering II. Relating muscle recruitment 
to fuel selection. J Appl Physiol 2004;96:41-49.

43. Janský L. Non-shivering thermogenesis and its thermoregulatory significance. Biol Rev Camb Philos Soc 1973;48:85-132.

44. Langeveld M, Tan CY, Soeters MR, et al. Mild cold effects on hunger, food intake, satiety and skin temperature in humans. Endocr Connect 2016;5:65-73.

45. Yoneshiro T, Aita S, Kawai Y, Iwanaga T, Saito M. Nonpungent capsaicin analogs (capsinoids) increase energy expenditure through the activation of brown adipose tissue in humans. Am J Clin Nutr 2012;95:845-50.

46. Cypess AM, Weiner LS, Roberts-Toler C, et al. Activation of Human Brown Adipose Tissue by a 33 -Adrenergic Receptor Agonist. Cell Metab 2015;21:3338.

47. U Din M, Saari T, Raiko J, et al. Postprandial Oxidative Metabolism of Human Brown Fat Indicates Thermogenesis. Cell Metab 2018:1-10.

48. Muzik O, Mangner TJ, Leonard WR, Kumar A, Janisse J, Granneman JG. $15 \mathrm{O}$ PET Measurement of Blood Flow and Oxygen Consumption in Cold-Activated Human Brown Fat. J Nucl Med 2013;54:523-531.

49. Ouellet V, Labbé SM, Blondin DP, et al. Brown adipose tissue oxidative metabolism contributes to energy expenditure during acute cold exposure in humans. J Clin Invest 2012;122:545-52.

50. Schilperoort M, Hoeke G, Kooijman S, Rensen PCN. Relevance of lipid metabolism for brown fat visualization and quantification. Curr Opin Lipidol 2016;27:242-248.

51. Oreskovich SM, Ong FJ, Ahmed BA, et al. MRI Reveals Human Brown Adipose 
Tissue Is Rapidly Activated in Response to Cold. J Endocr Soc 2019;3:23742384.

52. Reber J, Willershäuser M, Karlas A, et al. Non-invasive Measurement of Brown Fat Metabolism Based on Optoacoustic Imaging of Hemoglobin Gradients. Cell Metab 2018;27:689-701.e4.

53. M V, Schrauwen P, Backes WH, et al. Imaging cold-activated brown adipose tissue using dynamic T2*-weighted magnetic resonance imaging and 2-deoxy-2[18F]fluoro-D-glucose positron emission tomography. Invest Radiol $2013 ; 48: 708-14$

54. Sanchez-Delgado G, Martinez-Tellez B, Garcia-Rivero Y, et al. Brown Adipose Tissue and Skeletal Muscle 18F-FDG Activity After a Personalized Cold Exposure Is Not Associated With Cold-Induced Thermogenesis and Nutrient Oxidation Rates in Young Healthy Adults. Front Physiol 2018;9:1577.

55. Yoneshiro T, Wang Q, Tajima K, et al. BCAA catabolism in brown fat controls energy homeostasis through SLC25A44. Nature 2019;572:614-619. 


\section{TABLES}

Table 1. Participants' characteristic.

Study 1 CIT Study 1 NUTox Confirmatory study
analyses $(\mathrm{n}=44) \quad$ analyses $(\mathrm{n}=18) \quad($ Study $2, \mathrm{n}=13)$

\begin{tabular}{lrlrl}
\hline Sex $(\mathrm{n}$ women. \%) & $29(65.9)$ & 13.0 & $(72.2)$ & $6.0(46.2)$ \\
Age (years) & $22.2(2.2)$ & $21.9(2.0)$ & $25.6(3.0)$ \\
BMI $\left(\mathrm{kg} / \mathrm{m}^{2}\right)$ & $25.6(5.3)$ & $24.3(4.6)$ & $23.6(2.4)$ \\
Lean mass $(\mathrm{Kg})$ & $42.7(10.4)$ & $40.4(8.0)$ & $45.7(13.3)$ \\
Fat mass $(\mathrm{Kg})$ & $27.2(10.6)$ & $25.0(9.6)$ & $18.4(3.8)$ \\
Fat mass percentage $(\%)$ & $37.0(8.0)$ & $36.1(7.0)$ & $28.4(6.6)$ \\
RMR (Kcal/day) & $1565(278)$ & $1554(227)$ & $1484(286)$ \\
RER & $0.862(0.054)$ & $0.842(0.048)$ & $0.833(0.036)$ \\
Cooling vest water temperature $\left({ }^{\circ} \mathrm{C}\right)$ & $8.4(3.5)$ & $9.2(3.8)$ & $12.1(3.1)$ \\
\hline
\end{tabular}

Data are means (standard deviation) except for sex. CIT: Cold-induced thermogenesis; NUTox:

Macronutrient oxidation rates; BMI: Body mass index; RMR: Resting Metabolic Rate; RER: resting respiratory exchange ratio. 


\section{FIGURE LEGENDS}

Figure 1. Participant's flow-chart. ACTIBATE: Activating brown adipose tissue through exercise; BMI: body mass index, CIT: Cold-induced thermogenesis; ECG: electrocardiogram; NUTox: Nutrient oxidation, RER: Respiratory exchange ratio.

Figure 2. Cold-induced thermogenesis (CIT) and macronutrient oxidation rates (study 1). Panels A (adapted from (33)) C and D: Minute 0 represents the resting metabolic rate (RMR). Values are mean and standard error of the mean. $\mathrm{P}$ values for the main effect of the repeated measures ANOVAs. * represent values significantly different than RMR. Panel B: histogram representing over-all CIT (each column representing one individual's CIT). Dashed line represents mean CIT. CHOox: carbohydrates oxidation; FATox: fat oxidation; Kcal: Kilocalories; min: minutes; RER: respiratory exchange ratio; $\mathrm{VO}_{2}$ : oxygen consumption; $\mathrm{VCO}_{2}$ : carbon dioxide production.

Figure 3. Cold-induced thermogenesis (CIT) and macronutrient oxidation rates (study 2). Panels A, C and D: Minute 0 represents the resting metabolic rate (RMR). Values are mean and standard error of the mean. $\mathrm{P}$ values for the main effect of the repeated measures ANOVAs. Panel B: histogram representing over-all CIT (each column representing one individual's CIT). Dashed line represents mean CIT. CHOox: carbohydrates oxidation; FATox: fat oxidation; Kcal: Kilocalories; min: minutes; RER: respiratory exchange ratio; $\mathrm{VO}_{2}$ : oxygen consumption; $\mathrm{VCO}_{2}$ : carbon dioxide production.

Figure 4. Protein oxidation before (10 hours) and during mild cold exposure in the study 2. P value is for paired t-test. 